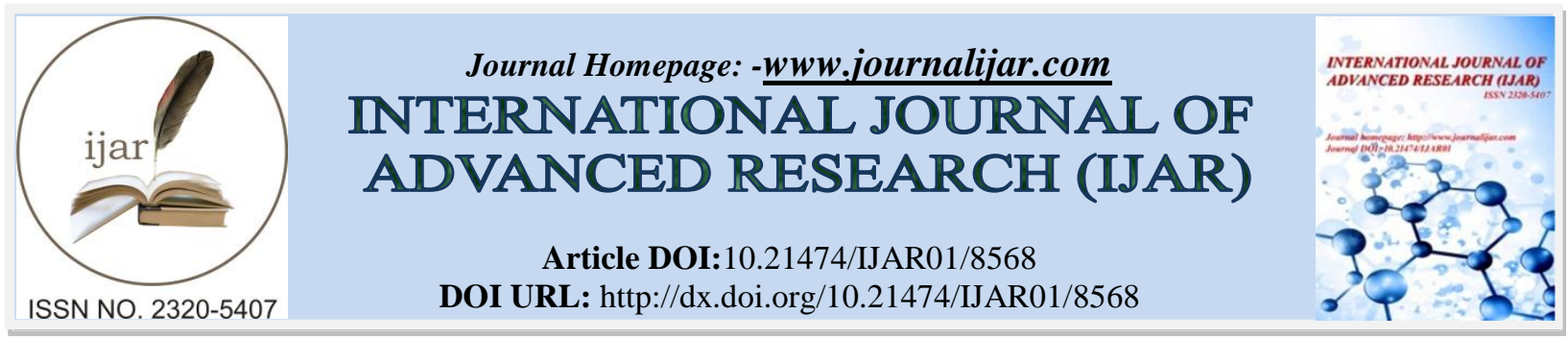

RESEARCH ARTICLE

\title{
PREVALENCE AND RISK FACTORS OF CAROTID ARTERY STENOSIS IN ISCHAEMIC STROKE PATIENTS- A CROSS SECTIONAL STUDY IN A TERTIARY CARE HOSPITAL.
}

\author{
Bharathikunisetty $^{1}$, Stephen ${ }^{1}$, Senthilnathan ${ }^{1}$, R.Deepak Arjundas ${ }^{2}$, Ayyar,S.S.K ${ }^{2}$ and Suresh Kumar ${ }^{2}$. \\ 1. Department of Neurology,Sree Balaji Medical College\&Hospital,Chrompet,Chennai,India. \\ 2. Department of Neurology,VijayaHealthCentre, Vadapalani,Chennai,India.
}

\section{Manuscript Info}

Manuscript History

Received: 14 December 2018

Final Accepted: 16 January 2019

Published: February 2019

\section{Key words:-}

carotid artery stenosis. Carotidintimal thickness,Doppler sonography .carotid end arterectomy.

\section{Abstract}

Background and purpose: Carotid artery stenosis is a major risk factor for stroke and for the symptomatic cerebrovascular disease. Approximately $20-30 \%$ of all ischaemic strokes are caused by carotid occlusive disease. In this study we attempted to find the prevalence of carotid artery stenosis in ischaemic stroke patients, so that aggressive secondary preventive measures can be directed to those patients. In this study we have analysed association between Carotid artery stenosis and risk factors such as Diabetes mellitus, Hypertension, Hyperlipidemia, Smoking, Sex and Age.

Methods: It is a cross sectional Hospital Based Prevalence Study done in a sample of 100 patients who were admitted in our hospital within 1 week of stroke during the period of july 2017 to june 2018. Patients whose duration of stroke $>2$ weeks, patients with haemorrhagic stroke, patients with H/o head injury and stroke due to infectious causes were excluded.

All patients were subjected to CT scan or MRI brain study and Colour Doppler study of extra cranial carotid arteries and vertebral arteries. Systolic and diastolic velocity of blood flow, carotid intimal medial thickness, presence of atheromatous plaque and thrombus was looked for and then the percentages of stenosis of the affected arteries were calculated .

Results:The percentage of patients with significant stenosis (>70\%) was $10 \%$ in our study. The prevalence of moderate stenosis was $12 \%$, mild stenosis was $24 \%$ and $54 \%$ of stroke patients had no Carotid stenosis. We found in our study the percentage of patients who had Carotid stenosis, increased with increase in age ${ }^{1}$. The prevalence in patients $<50$ years, $50-69$ years, $>60$ years was $30 \%$, 43\%, and $53 \%$ respectively. The majority (53\%) of patients with carotid artery stenosis were older than 60 years. Hypertension was the most common risk factor present in 54\% of cases either as a single risk factor or associated with other risk factors. The prevalence of carotid stenosis was more in hypertensive's $(57 \%)$ than in normotensives $(32 \%)$. Elevated systolic blood pressure accelerates the progression of intima medial thickness (IMT) in the carotid artery. However isolated hypertension occurs in only less than $20 \%$ of patients with stroke and is usually associated

Corresponding Author:-Bharathikunisetty. 
with other risk factors that is why antihypertensive treatment alone may fail to prevent stroke. We have found that age, male sex, smoking, HT, DM and Hyperlipidemia is associated with increased rate of carotid stenosis. In our study every patient with carotid artery stenosis had one or the other risk factor for carotid atherosclerosis. In other words, there was no patient with carotid artery stenosis, without any risk factor in our study. Hence asymptomatic patients with these risk factors should be screened for carotid stenosis to prevent stroke.

Conclusions- There was no patient with carotid artery stenosis, without any risk factor in our study. Hence asymptomatic patients with these risk factors should be screened for carotid stenosis to prevent stroke . About $46 \%$ of ischaemic stroke patients had carotid stenosis in our study.

The prevalence of carotid stenosis increases with increase in age, male gender, smoking, DM, HT \& Hyper lipidemia. Carotid stenosis and if present, should have their blood glucose, blood pressure and lipids under control and should be started on antiplatelet drugs and statins for plaque regression and for primary prevention and recurrences of stroke. Carotid endarterectomy should be done in selected cases for secondary prevention of stroke. The present study highlights the importance of Doppler sonography in stroke prevention effort through surveillance for atherosclerosis that predispose a person to cerebral ischaemia.

Copy Right, IJAR, 2019,. All rights reserved.

\section{Introduction:-}

Cerebrovascular accident is an important health problem worldwide ${ }^{2}$. The annual incidence of stroke is 0.2 to 2.5/1000 population. Worldwide approximately 20 million suffer from stroke each year, of these 15 million survive, while the other 5 million become disabled by stroke ${ }^{3}$. In India, incidence of stroke is 33/1,00,000 population ${ }^{4}$. Analysis of community surveys from different regions of India showed a crude stroke prevalence rate of about 203 per 100,000 populations above 20 years of age, amounting to a total of about 1 million cases. The male-to-female ratio was estimated to be 1.7 . However, a recently reported community survey in Kolkata revealed a stroke prevalence rate of 545 per 100,000 population, which is equal to or higher than that reported from developed countries. Clinically stroke is the result of a disturbance of cerebral circulation, either due to occlusion of main blood vessel due to thrombo-embolism or rupture of a blood vessel. About $85 \%$ of all strokes are of ischemic origin, caused by thrombotic or embolic blockage of a cerebral artery. Ischemic strokes occurring in the anterior circulation are the most common of all ischemic strokes, accounting for approximately $70 \%$ of all cases. They are caused most commonly by occlusion of one of the major intracranial arteries or of the small single perforator (penetrator) arteries. The most common causes of arterial occlusion involving the major cerebral arteries are (1) emboli, most commonly arising from atherosclerotic arterial narrowing at the bifurcation of the common carotid artery, from cardiac sources, or from atheroma in the aortic arch and (2) a combination of atherosclerotic stenosis and superimposed thrombosis. Carotid stenosis is being assessed by PSV (peak systolic velocity) and EDV (end diastolic velocity) of blood flow in carotid arteries. A mild stenosis (up to 50\%) is characterized by local increase of peak and mean flow velocities, moderate stenosis (50-69\%) shows a distortion of the normal pulsatile flow in addition to local increase of peak and means frequencies. Peak systolic flow deceleration is found in the post stenotic segment and a severe stenosis $(>70 \%)$ produces marked increase in peak velocities and a subtotal stenosis $(>95 \%)$ is characterized by a small signal of variable frequencies that decrease once a stenosis becomes pseudo occlusive.

\section{Methods:-}

A Cross sectional Hospital Based Prevalence Study was conducted in Sree Balaji Medical College and Hospital, Chromepet, Chennai during the year July2017 to July 2018. It includes 100 patients, aged 18-80 yrs, of both sexes with acute stroke duration less than 2 weeks, with CT brain showing infarct. Patients with duration of stroke $>2$ weeks, with Haemorrhagic stroke, with H/o head injury and with serious systemic illness, stroke due to infections, malignancy were excluded. All patients were examined clinically and detailed history was noted and blood investigations like $\mathrm{Hb} \%$, TC, DC, ESR, platelet count, Fasting blood sugar, urea, Serum Creatinine, fasting lipid 
profile, urine analyses, and CXR, ECG, Echo were done for all patients. All patients were subjected to CT scan brain study and colour Doppler study of extra cranial carotid arteries and vertebral arteries. Systolic and diastolic velocity of blood flow, carotid intimal medial thickness, presence of atheromatous plaque and thrombus was looked for and then the percentages of stenosis of the affected arteries were calculated.

\title{
Statistical Analysis:-
}

Statistical analysis was carried out for 100 subjects. Age, Presence of diabetes, hypertension, Smoking, Alcoholism and hyperlipidemia were analyzed in patients with and without carotid stenosis admitted for acute ischemic stroke. The statistical significance was calculated using Chi- square test. Statistical significance was taken when P value was $<0.05$. Statistical analysis was carried using standard formulae. Microsoft Excel 2007 and SPSS (Statistical Package for Social Sciences software's) were used for data entry and analysis.

\section{DISTRIBUTION OF SAMPLE BY CAROTID STENOSIS}

\author{
$\square$ NO STENOSIS $\square$ STENOSIS
}

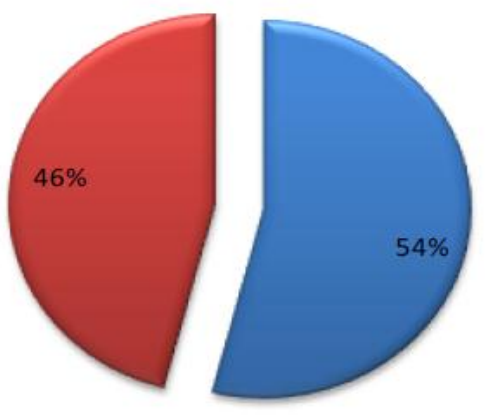

It is found that the percentage of patients with carotid stenosis was increasing with increase in age.

\section{FREQUENCY OF CAROTID STENOSIS}

\section{IN HT}

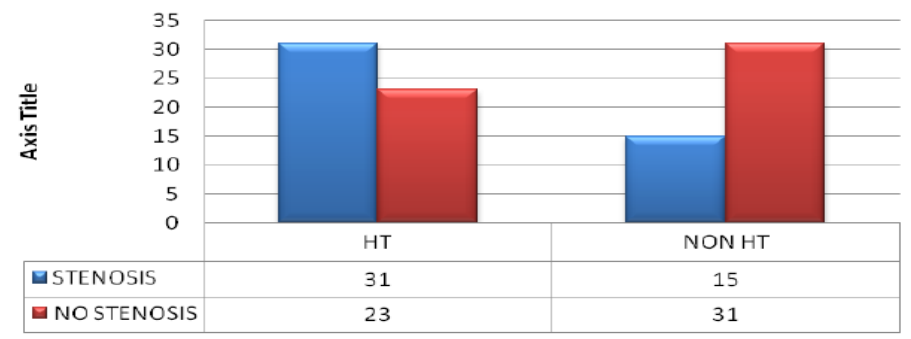

54 patients had hypertension in our study in which 31 of them had carotid artery stenosis and 46 of them were normotensives in whom 15 of them had carotid artery stenosis. Frequency of Carotid artery stenosis was more in Hypertensive's than in normotensives and it was statistically significant. 


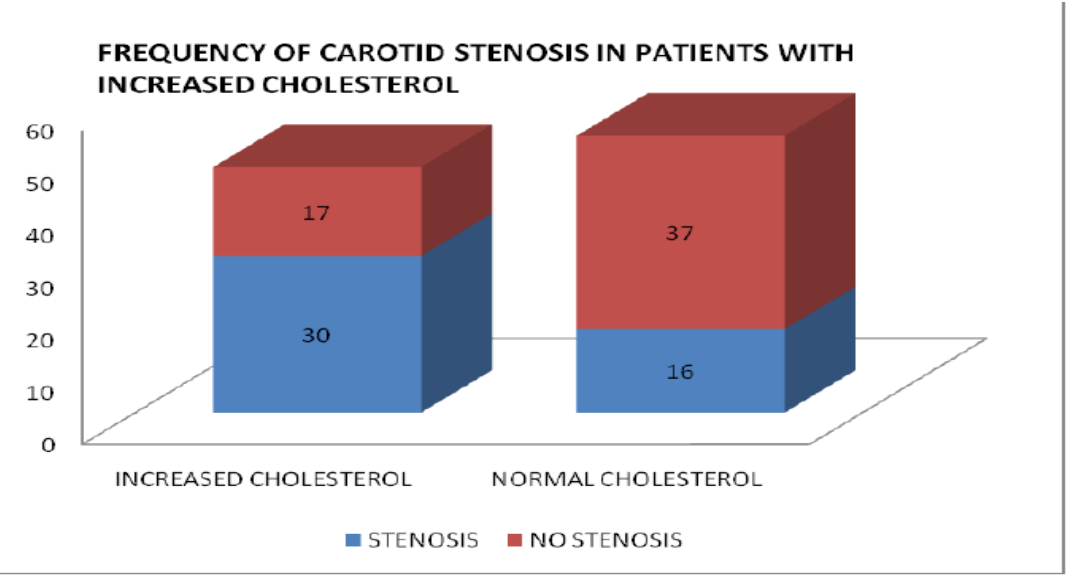

47 patients from the sample had increased level of total cholesterol in which 30 of them had carotid artery stenosis and 53 of the patients had total cholesterol $<200 \mathrm{mg} / \mathrm{dl}$ in which 16 of them had carotid artery stenosis indicating frequency of Carotid artery stenosis was more in Patients with raised total Cholesterol than in patients with normal total Cholesterol and it was statistically significant.80 patients had HDL > 40 in our study in which 30 of them had carotid artery stenosis and 20 of them were HDL $<40$ in whom 16 of them had carotid artery stenosis. Frequency of Carotid artery stenosis was more in Patients with Low HDL than in patients with Increased HDL and it was statistically significant

\section{PREVALANCE OF CAROTID STENOSIS IN PATIENTS WITH RAISED TGL}

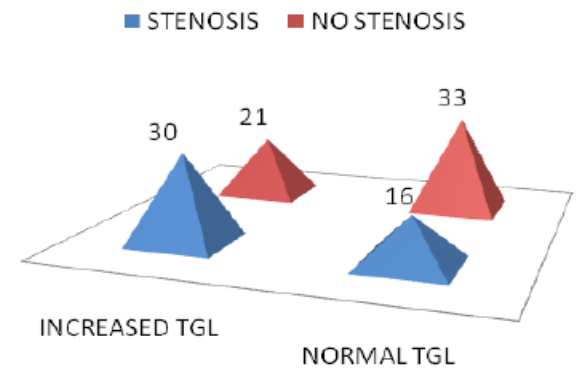

\section{DISTRIBUTION OF SAMPLE BASED ON SEVERITY OF CAROTID STENOSIS}

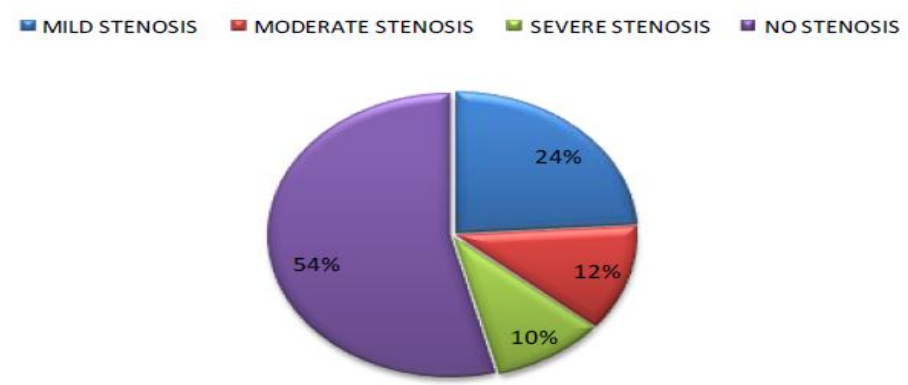

In our study 54 of them did not have carotid artery stenosis. Out of 46 who had carotid artery stenosis $24,12,10$ of them had mild, moderate severe stenosis respectively. There was a marginal increase in Left side Carotid stenosis. It was found that Carotid artery stenosis was more on Common Carotid arteries than Internal Carotid arteries. 


\section{Discussion:-}

In our study we have found that the prevalence of carotid stenosis in acute Ischaemic stroke patients is about $46 \%$, consistent with studies done by Olivieroet $\mathrm{al}^{5}$. In their study the prevalence of carotid stenosis was about $43 \%$ in Ischaemic stroke patients. In our study the percentage of patients with significant stenosis $(>70 \%)$ was $10 \%$.The prevalence of significant stenosis in a study conducted by M.M.Singh et al was about $32 \%$. In our study the prevalence of moderate stenosis was $12 \%$, mild stenosis was $24 \%$ and $54 \%$ of stroke patients had no Carotid stenosis. The prevalence of asymptomatic carotid stenosis $(>50 \%)$ in a study conducted in asymptomatic carotidstenosis patients by P.P.Mineva et al was $6.4 \%{ }^{7}$.

\section{Age And Carotid Stenosis:-}

Older age is an important and well known risk factor for the development of Carotid artery atherosclerosis. In our study the majority $(53 \%)$ of patients with carotid artery stenosis were older than 60 years. We found in our study the percentage of patients who had Carotid stenosis, increased with increase in age ${ }^{1}$. The prevalence in patients $<50$ years, 50-69 years, >60 years was 30\%, 43\%, and 53\% respectively. Carotid stenosis in keeping with Atherosclerotic diseases increases with age. The risk of Carotid Atherosclerosis increases after 45 years of age.

\section{Sex And Carotid Stenosis:-}

In our study we found that the prevalence of carotid stenosis was more in Males (46\%) than Females (44\%).The majority of our patients with carotid artery stenosis were male but this gender difference was found to be not significant, but studies conducted by Jacob et $\mathrm{al}^{8}$ and by Ralph et $\mathrm{al}^{9}$ shows that Carotid stenosis was commoner in males than females.

\section{Risk Factors Of Carotid Atherosclerosis:- Diabetes Mellitus and Carotid Stenosis:-}

Atherosclerosis is presumed to be accelerated in diabetes for a number of reasons. First, diabetes is associated with an increased risk of traditional coronary heart disease (CHD) risk factors, including hypertension, dyslipidaemia, obesity, and hyperinsulinaemia, other metabolic disturbances unique to diabetes, such as increased levels of circulating glucose, advanced glycation end products, and oxidation of lipoproteins might also increase the risk and rate of atherosclerosis. Carotid artery stenosis was common in diabetics (64\%) than non-diabetics (32\%) and it was statistically significant. K.Rajamaniet al ${ }^{1}$ have shown in their study that carotid stenosis was common in diabetics.

\section{Hypertension and Carotid Stenosis:-}

A number of studies have demonstrated that hypertension is an important risk factor in stroke. The Framingham Study, confirmed by other population studies, determined that systolic BP is a better predictor than diastolic BP of the risk of atherothrombotic brain infarction. Hypertension was the most common risk factor present in 54\% of cases either as a single risk factor or associated with other risk factors. In our study we found that hypertension was one of the risk factors for carotid stenosis and the prevalence of carotid stenosis was more in hypertensive's (57\%) than in normotensives $(32 \%)$ consistent with studies done by Duncan et al ${ }^{10}$, Sutton et $\mathrm{al}^{11}$. They, in their study, found that asymptomatic carotid stenosis was found in $25 \%$ of adults with hypertension, than those without Hypertension. The predictors of carotid stenosis were systolic BP > $160 \mathrm{mmHg}$ and in isolated Systolic Hypertension patients when Diastolic BP was $<75 \mathrm{mmHg}$ there was a strong correlation with carotid stenosis. Elevated systolic blood pressure accelerates the progression of intima medial thickness (IMT) in the carotid artery, However isolated hypertension occurs in only less than $20 \%$ of patients with stroke and is usually associated with other risk factors that is why antihypertensive treatment alone may fail to prevent stroke.

\section{Smoking and Carotid Stenosis:-}

Smoking is associated with raised fibrinogen levels, increased packed cell volume, and decreased macrophage activity changes in lipid biochemistry. Smoking increases arterial wall stiffness and alters the pattern of arterial blood flow. In our study we found that smoking acts as a risk factor for carotid stenosis. More smokers (66\%) had carotid stenosis than Non-smokers (32\%), which is also shown by H.R. Muller et al $^{12}$. Smoking as a risk factor for carotid stenosis.

\section{Hyperlipidemia and carotid stenosis:-}

Higher LDL cholesterol levels are associated with higher incidence of carotid atherosclerotic disease while high levels of HDL cholesterol have protective role. In our study the prevalence of patients with increased Cholesterol (> $200 \mathrm{mg} / \mathrm{dl})$, increased TGL $>>150 \mathrm{mg} / \mathrm{dl})$, decreased HDL $(<40 \mathrm{mg} / \mathrm{dl})$ and increased LDL $(>130 \mathrm{mg} / \mathrm{dl}) \mathrm{were} 64 \%$, 
$59 \%, 80 \%$ and $67 \%$ respectively. The prevalence in patients with decreased cholesterol $(<200 \mathrm{mg} / \mathrm{dl}), \mathrm{decreased}$ TGL (<150mg/dl), Increase HDL (>40mg/dl) and decreased LDL (<130mg/dl) were 30\%, 32\%, 37\% and 31\% respectively. Prevalence of carotid stenosis, just like coronary atherosclerotic disease, increases with Hyper cholesterolemia (>200mg/dl) and Increased LDL (> 150mg/dl) and Increased TGL (>130mg/dl) and decreased HDL $(<40 \mathrm{mg} / \mathrm{dl})$. They are associated with extra cranial large vessel atherosclerosis and also coronary atherosclerosis. Carotid atherosclerosis leads to increase in IMT and plaque formation and stenosis ${ }^{13}$. Extra cranial carotid atherosclerosis is associated with major brain vessel occlusion, leading to infarct of brain tissue.

\section{Site and carotid stenosis:-}

In our study carotid stenosis was found at the bifurcation of CCA and the origin of ICA. Carotid stenosis was more on left Side and was more on CCA than ICA. CCA stenosis was found in 59\% of patients and ICA stenosis was found in $41 \%$ of patients. We have found that age, male sex, smoking, HT, DM and Hyperlipidemia are associated with increased rate of carotid stenosis. In our study every patient with carotid artery stenosis had one or the other risk factor for carotid atherosclerosis. In other words, there was no patient with carotid artery stenosis, without any risk factor in our study. Hence asymptomatic patients with these risk factors should be screened for carotid stenosis to prevent stroke.

\section{Limitations:-}

The present study has some limitations. As this study was a single hospital-based study conducted on different clinical and risk factor profiles, these results cannot be applied to the general population. As Doppler sonography was performed by more than one sonographer so an observer bias in categorization of the carotid stenosis could not be ruled out.

\section{Conclusion:-}

100 Patients with acute ischemic stroke patients with CT or MRI Brain showing infarcts were evaluated .In our study age, sex, history of DM, HT, Smoking, FBS and Fasting lipid profile were recorded for the subjects. They were subjected to Doppler Ultrasonography of the carotid arteries which is a cost effective and non-invasive method to detect atherosclerotic plaque, carotid stenosis and to measure the degree of stenosis. The prevalence of carotid stenosis in our study was $46 \%$. The prevalence of mild, moderate and severe stenosis were $24 \%, 12 \%$ and $10 \%$ respectively correlating with other studies ${ }^{14}$. The distribution of carotid stenosis was more on left side and CCA $(59 \%)$ was the common site of atherosclerotic plaque and stenosis. There was a statistically significant correlation between increasing age, smoking, DM, HT, and Hyperlipidemia and the prevalence of carotid stenosis correlating with other studies.

\section{Summary:-}

Carotid stenosis is one of the common causes of ischaemic stroke. About $46 \%$ of ischaemic stroke patients had carotid stenosis in our study. The prevalence of carotid stenosis increases with increase in age, male gender, smoking, DM, HT \& Hyper lipidemia. DM, HT, Smoking \& Hyperlipidemia act as risk factors for carotid stenosis. Hence patients with DM, HT \&Hyperlipidemias should have their carotid arteries screened to detect asymptomatic carotid stenosis and if present, should have their blood glucose, blood pressure and lipids under control and should be started on antiplatelet drugs and statins for plaque regression and for primary prevention of stroke. Patients with stroke who have carotid stenosis (symptomatic carotid stenosis) are prone for recurrence of stroke. They should be advised to control the risk factors for carotid stenosis and should be started on anti platelet drugs and statins. Carotid endarterectomy should be done in selected cases for secondary prevention of stroke ${ }^{15}$.

The present study highlights the importance of Doppler sonography in stroke prevention effort through surveillance for atherosclerosis that predispose a person to cerebral ischaemia. Doppler sonography of the carotid arteries in high risk individuals could therefore have profound diagnostic and therapeutic implications in predicting and preventing a potentially fatal and devastating stroke.

\section{Bibilography:-}

1. K. Rajamani et al. Carotid stenosis in African-American men. Journal of Vascular Surgery. Vol.43, 1162-1165.

2. Park's textbook of preventive and social medicine 20th ed. Jabalpur banavideBhanot publishers 1994.

3. Mc Mohan S Introduction the global burden of stroke in Chalmess J editor Clinicians manual of blood pressure and stroke prevention science press London, 2002. 
4. Prasad K Recent concepts in stroke in Bansal BC Agarwal AK Epidemiology of cerebrovascular disease in India, Mumbai. Indian college of Physicians: 1999 pp 11-19.

5. Oliviero U, Orefice G, Coppola G, Scherillo G, Ascione S, Casaburi C, Barbieri F, Sacca L. Carotid atherosclerosis in ischaemic stroke patients. Int. Journal of Angiology. 2002 Jun;21(2):117-22.

6. M.M. Singh, S. Gupta et al. Carotid stenosis in Stroke, JAPI-1996; Vol.44 No.12. 954-956.

7. P.P.Mineva et al: Prevalence and outcome of asymptomatic carotid stenosis: A population based ultrasonographic study: European Journal of Neurology: July 2002: Vol-9: Issue-4: 383-388.

8. Jacob Selhub et al. Association between Homocysteine\& Carotid stenosis. NEJM, 1995, Vol.333. Page 325.

9. Sacco RL, Mohr JP: Infarcts of undetermined cause: NINCDS Stroke Data Bank: Ann of Neurology, 1989: 25; 382-90.

10. Duncan GW, Lees RS, Ojemann R, David SS: Concomitants of atherosclerotic carotid artery stenosis. Stroke 1977; 8:665-669

11. Sutton KC, Wolfson SK Jr, Kuller LH: Carotid and lower extremity arterial disease in elderly adults with isolated systolic hypertension. Stroke 1987;18:817-822.

12. H.R. Muller et al: Smoking and carotid stenosis: Journal of Neurology: 1990: 97-102.

13. Glagov et al: Hemodynamics and atherosclerosis: Insides and perspectives gained from studies of human arteries: Arch Pathology. 1998: 112: 1018-1031.

14. Kaul S, Alladi S, Mridula KR, Bandaru VC, Boddu DB, Anjanikumar D, et al. Prevalence and risk factors of carotid intima-media thickness in asymptomatic individual subjects in a tertiary care center in India. Ann Indian AcadNeurol 2015;18:430-4.

15. Abbott AL. Medical (nonsurgical) intervention alone is now best for prevention of stroke associated with asymptomatic severe carotid stenosis: Results of a systematic review and analysis. Stroke 2009;40:e573-83. 